\title{
Russian Health-related Quality of Life Assessment According to Comq-12 in Patients with Different Forms of Chronic Otitis Media
}

\author{
S. la. Kosyakov, J. V. Minavnina \\ Department of Otorhinolaryngology \\ Russian Medical Academy of Postgraduate Education, Moscow, Russia, 125993
}

Running head: Russian health-related quality of life assessment Abbreviations:

$\mathrm{COM}$ - chronic otitis media

COMQ-12 - chronic otitis media questionnaire-12

HRQoL - health-related quality of life

RCOMQ-12 - Russian version of the chronic otitis media questionnaire-12

Key-words: chronic otitis media; quality of life; questionnaire; chronic disease; surveys and questionnaires; translations

Source of financial support: none.

\section{Abstract.}

Background: Chronic otitis media is a widespread disease that affects up to $2 \%$ of the population. The Chronic Otitis Media Questionnaire12 was developed to assess the patient-reported health-related quality of life reduction due to chronic otitis media. The aim of this study is to evaluate the characteristics of the chronic otitis media questionnaire12 among various groups of chronic otitis media patients.

Materials and methods: The Russian version of the questionnaire was obtained through translation and back-translation. 108 patients with different forms of COM completed the Russian version of the questionnaire. The internal consistency of this questionnaire was evaluated using Cronbach's alpha coefficient.

Results: The average score was 19.4 (standard deviation 8.3). The internal consistency of the Russian version of the questionnaire was high, with a Cronbach's alpha value of 0.860 .

Conclusions: Russian version of the Chronic Otitis Media Questionnaire-12 is a reliable tool for assessment of health-related quality of life in chronic otitis media patients. It can be used in the future to determine a plan of surgical treatment and forecasting of results.

\section{Corresponding autor:}

Sergey Kosyakov, M.D., Ph.D.

Russian Medical Academy of Postgraduate Education Department of otorhinolaryngology

Ivankovskoye street, 7

125993, Moscow, Russia

E-mail: serkosykov@yandex.ru

\section{Introduction}

Chronic otitis media (COM) is a widespread disease that affects up to $2 \%$ of the population [1]. There are several forms of COM, each of which is characterized by peculiarities of courses and outcomes. Some forms of COM, due to their asymptomatic courses, do not to affect the patient for a long period of time, and, therefore, often remain undiagnosed and can lead to a number of complications. Other forms of COM occur with a vivid clinical picture expressed by the complaints of the patient and decrease their quality of life. Patients of the second group are much more likely to seek medical care, require active treatment tactics and may well have high, possibly unrealistic expectations of the treatment. It should be noted that the symptoms can be exaggerated, and patient's expectations can be much greater than the forecasts of the surgeon, based on physical examination data. In such situations an objective tool is required to evaluate the relationship between pattern of disease perceived by the patient and pattern of disease, based on objective data. Another objective of the surgery is the need to meet the expectations of the patient. Inevitably, perfectly executed surgery does not always provide a corresponding improvement in the quality of life of the patient. A connecting element between the expectations of the patient and the surgeon's capabilities has recently developed the use of the health-related quality of life (HRQoL) questionnaires. The chronic otitis media 
questionnaire-12 (COMQ-12) was devised to assess the patient-reported reduction of quality of life due to COM [2]. This questionnaire can also determine the need for treatment and prognosis of surgery. The original English version of the COMQ-12 was developed by Phillips et al. [2, 3]. The aim of this study is to evaluate the characteristics of the Russian version of the COMQ-12 (RCOMQ-12) among various groups of COM patients.

\section{Materials and methods}

Russian version of the COMQ-12, as well as the original English version consists of 12 questions characterizing various aspects of the COM patients' condition, each of which can be assessed on a five point scale (appendix 1). 8 questions $(1-7,12)$ describe the severity of the disease, whereas the remaining 4 questions describe frequency of their occurrence. We made a validation of the Russian version of the COMQ-12 with authors and obtained permission for its use. The study included 108 patients with a history of COM, distributed into groups depending on the form of the disease who were asked to fill out a Russian version of the COMQ-12.

\section{Results}

The study included 108 patients: 49 men (45\%) and 59 women (55\%), ranging in age from 16 to 84 years. Due to the various forms of COM and previous surgery we identified 4 groups of patients: group A - perforated eardrum - 58 patients (54\%), group B - cholesteatoma - 21 patients $(19,4 \%)$, group $\mathrm{C}$ - patients after previous surgery (open mastoid cavity) - 18 patients $(16,6 \%)$ and group D - patients with adhesives otitis media and fibrosis - 11 (10\%). COMQ-12 scores ranged from 4 to 43 among all respondents out of a possible 60 points. The average score was 19.4 (SD 8.3). The median COMQ-12 score overall was 20, and the modal score was $14.91 \%$ of respondents achieved a score of 30 or less, $55 \%$ of respondents achieved a score of 20 or less out of a possible 60 points.

A detailed analysis of the groups showed us the following results: in the group A -scores from 4 to 43 (18.5 in average), in the group B - from 6 to 41 (21.2 in average), in the group $\mathrm{C}$ - from 5 to 37 (21.8 in average), in the group D - ranging from 5 to 28 (16.6 in average). In the Russian version of the COMQ-12 Cronbach's alpha is equal to 0.860 .

\section{Discussion}

COMQ-12 was developed to assess the impact of disease on HRQoL from the patients perspective [2]. In the Russian version of the COMQ12 Cronbach's alpha is equal to 0.860 , which confirms the high internal consistency of the questionnaire and a high degree of correlation between its separate points. For comparison, Cronbach's alpha version in a Dutch study is 0.833 , in the original English version - 0.889 [2, 4]. This index is used to measure the reliability of test scores: a factor greater or equal to 0.70 is defined as acceptable, equal to 0.80 or more as good.

To adequately interpret the COMQ-12 scores, we must have the reference points which are typical for a healthy population without COM. A study by Phillips et al. determined normal values for the COMQ-12 scores in an adult population without active COM [3]. We did the same study on our population. Sixty healthy volunteers without COM were asked to complete Russian version of COMQ12.

COMQ-12 scores overall ranged from 0 to 14 among all respondents with a mean score of 3.55. Among patients without complaints average score is 2.07 (range $0-8$ ), among patients with complaints - 4.78 (range 0-14). The median COMQ-12 score overall was 2.5 and the modal score was 0 with 19 (31.7\%) participants achieving this score. $75 \%$ of respondents achieved a score of 5 or less, $95 \%$ of respondents achieved a score of 10 or less out of a possible maximum of 60 .

The results of both studies show that 5 scores of less can be a characteristic of the healthy population.

The present study is another step in the development of the Russian version of COMQ-12 and covers only the preoperative data of patients. Postoperative data and surgical selection will be the subject of our further research.

\section{Conclusion}

In conclusion, Russian version of the COMQ-12 is a reliable tool for assessment of HRQoL in COM patients. The scores of the COMQ-12 vary among different groups of COM people. So, it can be used in the future to determine a plan of surgical treatment and forecasting of results. 
The authors declare that they have no conflict of interest.
The authors declare that they have nothing to disclose.

Letter to the Editors: I, Sergey Kosyakov, doctor in medicine and corresponding author of this manuscript, certify that all the co-workers have actively participated to the conception and the writing of this prospective study. This text is original and no part of it is under consideration for publication in another journal. We hope that it can be of some interest for the readers and request that you consider it for publication in your journal.

Sincerely yours,

Pr Sergey Kosyakov

\section{AUTHORSHIP CONTRIBUTION}

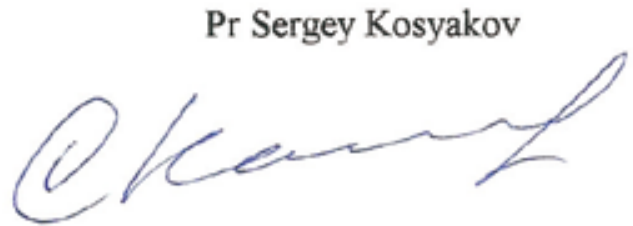

All the authors have contributed to the care of the patients. Sergey Kosyakov performed the operations described. All authors were involved in the literature research and the writing of the manuscript. The manuscript was edited by Sergey Kosyakov.

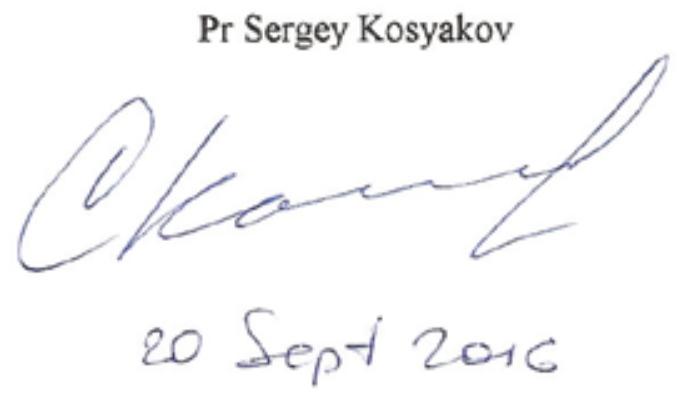

\section{References.}

1. Nadol J, Staecker H, Gliklich R. Outcomes Assessment for Chronic Otitis Media: The Chronic Ear Survey. The Laryngoscope. 2000; 110(S94): 32-35. doi:10.1097/00005537-200003002-00009.

2. Phillips J, Haggard M, Yung M. A New Health-Related Quality of Life Measure for Active Chronic Otitis Media (COMQ-12). Otology \& Neurotology. 2014; 35(3): 454-458. doi:10.1097/mao.0000000000000205.

3. Phillips J, Yung M. COMQ-12 scores in adult patients without chronic middle ear disease. Clinical Otolaryngology. 2014;39(6):362-367. doi:10.1111/coa.12306.

4. Oorts E, Phillips JS, Van de Heyning P, Yung M, Van Rompaey V. Development of the Dutch version of the health-related quality of life measure for chronic otitis media (COMQ-12). B-ENT 2015; 11(4): 291-295. 